\title{
Diagnóstico espacio temporal de las variables abióticas y bióticas, que afectan la estructura de la comunidad de los macroinvertebrados bentónicos, presentes en un ecosistema lótico
}

\author{
Diagnosis of temporary space abiotic and biotic variables that affect the community \\ structure of benthic, macroinvertebrates present in a river ecosystem
}

\section{Diagnóstico espaço temporário de variáveis bióticas e abióticas que afetam a estrutura da comunidade de macroinvertebrados bentônicos, presentes em um ecossistema fluvial}

\author{
Judith Yamile Ortega-Contreras ${ }^{1}$, Mabel Lorena Botello-Botello
}

Forma de citar: J.Y. Ortega-Contreras y M.L. Botello-Botello, "Diagnóstico espacio temporal de las variables abióticas y bióticas, que afectan la estructura de la comunidad de los macroinvertebrados bentónicos, presentes en un ecosistema lótico", Respuestas, vol. 22, no. 1, pp. 112-128, 2017.

Recibido:

Junio 22 de 2016

112

Aceptado:

Noviembre 16

de 2016
${ }^{1}$ M.Sc Ingeniería Ambiental judithyamileoc@ufps.edu.co Orcid: $0000-0002-8717-434 \mathrm{X}$ Grupo de investigación ambiente y vida GIAV Universidad Francisco de Paula Santander Cúcuta-Colombia

${ }^{2}$ Ingeniera Ambiental en ejercicio Semillero de Ciencia y Tecnología Ambiental SICTA Orcid: 0000-0001-2831-1900 Universidad Francisco de Paula Santander Cúcuta-Colombia

\section{Resumen}

Para determinar los cambios espacio temporales de las variables bióticas y abióticas que modifican la estructura de los macroinvertebrados bentónicos, se establecieron 6 estaciones de monitoreo distribuidas en el trayecto de la parte alta (Municipio de Pamplona -media (Municipio de Cúcuta)) del río Pamplonita. El muestreo se realizó durante 7 meses, para un total de 42 muestras distribuidas durante los periodos bimodales de lluvia y sequía. El tipo de investigación fue descriptiva y aplicada. Se obtuvieron los índices de contaminación por materia orgánica (ICOMO), Índice de Contaminación por Mineralización (ICOMI) e Índice de Contaminación por $\mathrm{pH}(\mathrm{ICOpH})$. La estructura de la comunidad bentónica se analizó mediante el índice de diversidad (Shannon-Weiner). Finalmente se validó el modelo matemático (Índice de Tolerancia frente un gradiente de contaminación por materia orgánica), a partir de las relaciones definidas entre las variables bióticas y abióticas. Los resultados del ICOMO reflejaron que la estación del terminal de Pamplona localizada a una altura de 2295 m.s.n.m., es la de mayor carga de materia orgánica con un promedio de 0,79, con un índice de diversidad de Shannon, promedio de 0,63, de diversidad muy baja. La estación de menor contaminación fue la del Puente de San Rafael, con un promedio de ICOMO igual a 0,44, y un índice de diversidad muy baja igual a 0,85.Los resultados biológicos registraron un total de 115 familias de macroinvertebrados acuáticos, 103 son organismos estenotípicos a condiciones de mala calidad del agua. Para la validación del modelo de índice de tolerancia, solo se aplicó a las 12 familias de mayor porcentaje de abundancia, consideradas euritípicas a la contaminación, ya que ésta situación se observó en las 6 estaciones de muestreo. Entre ellas las que reportaron mayor abundancia fueron Baetidae con $33 \%$ de abundancia, seguido de la familia Glossiphoniidae con 16,8\% de abundancia.

Palabras clave: Abiótico, biótico, calidad del agua, índice de contaminación, índice de diversidad, macroinvertebrados bentónicos. 
Abstract

To determine the spatial and temporal changes of the biotic and abiotic variables that modify the structure of the benthic macroinvertebrates, six monitoring stations were set up along the Upper Pamplona (Pamplona Municipality) of the Pamplonita River. Sampling was carried out during 7 months, for a total of 42 samples distributed during the bimodal times of rain and drought. The type of investigation was descriptive and applied. The indices of contamination by organic matter (ICOMO), Pollution Index by The structure of the benthic community was analyzed using the diversity index (ShannonWeiner) and finally the mathematical model was validated (Index of Tolerance against a gradient of contamination by matter The results of ICOMO showed that the terminal station of Pamplona located at a height of 2,295 msnm, is the one with the highest organic matter load with an average of 0,79 , with a Shannon diversity index, average of 0.63 , of very low diversity. The lowest contamination station was the San Rafael Bridge, with an ICOMO average of 0.44 , and a very low diversity index of 0.85 . The biological results recorded a total of 115 families of aquatic macro invertebrates, 103 arestenotypic organismsin conditions of poor water quality. For the validation of the tolerance index model, it was only applied to the 12 families with the highest percentage of abundance considered eurythyics to the contamination, since this situation was observed in the 6 sampling stations. Among them, Baetidae had a higher abundance with 33\% abundance, followed by the family glossiphoniidae with $16.8 \%$ abundance

Keywords: Abiotic, biotic, water quality, pollution index, diversity index, benthic macroinvertebrates.

\section{Resumo}

Para determinar as mudanças espaço-temporais de variáveis bióticas e abióticas que modificam a estrutura de macroinvertebrados, 6 estações de monitoramento distribuído no caminho do topo (Município -Media Pamplona (Município Cúcuta)) estabeleceu rio Pamplonita . A amostragem foi levada a cabo durante 7 meses, durante um total de 42 amostras distribuídas ao longo dos períodos bimodais de chuva e seca. A pesquisa foi descritiva e aplicada. os níveis de poluição obtidos por matéria orgânica (como deveria), Índice incrustantes para a mineralização (ICOMI) e Índice de proliferação de $\mathrm{pH}(\mathrm{ICOpH})$. A estrutura da comunidade bêntico foi analisada pelo índice de diversidade (Shannon-Weiner). Finalmente, o modelo matemático (índice de tolerância poluição orgânica gradiente) foi validada a partir das relações definidas entre as variáveis bióticos e abióticos. resultados ICOMO reflectida a estação terminal Pamplona localizado a uma altura de 2295 metros, é a mais elevada carga de matéria orgânica com uma média de 0,79, com diversidade de Shannon, média de 0,63, diversidade muito baixa. Estação menos poluição era Ponte San Rafael, com média de ICOMO igual a 0,44, e muito baixo índice de diversidade igual a 0,85.Los resultados biológicos relatou um total de 115 famílias de macroinvertebrados, 103 são estenotípicos organismos para condições de má qualidade da água. Para validar o índice de tolerância modelo, apenas as 12 famílias de maior percentual abundância, contaminação euritípicas considerado foi aplicado, como esta situação foi observada em seis estações de amostragem. Eles estão incluindo aqueles que relataram maior abundância foram Baetidae com 33\% de abundância, seguido por 16,8\% de família Glossiphoniidae com abundância.

Palavras-chave: Abióticos, bióticos, qualidade da água, índice de poluição, índice de diversidade, macroinvertebrados bentônicos.
ISSN 0122-820X

E-ISSN 2422-5053

PP: $112-128$ 
No. 1

Enero - Junio 2017

ISSN 0122-820X

E-ISSN 2422-5053

PP: $112-128$

114

\section{Introducción}

Ciertos ecosistemas cumplen la importante función de sumidero o vertedero, en los cuales se descargan ciertos desechos que son, en alguna medida, asimilados [1].

El Río Pamplonita, es un ecosistema estratégico que aporta bienes y servicios ambientales a la población de Norte de Santander, no obstante, es el mayor receptor de aguas residuales; el mayor caudal de efluentes de aguas negras domésticas lo recibe en su paso por las zonas urbanas de las ciudades de Cúcuta y Pamplona. Estas descargas afectan y modifican sus características ecológicas naturales. Adicionalmente, altera la composición, estructuran y funcionalidad del sistema acuático, disminuye la oferta hídrica ocasionando condiciones muy altas del índice de vulnerabilidad para el abastecimiento del recurso hídrico de la población que la utiliza como fuente de aprovisionamiento de agua.

La contaminación orgánica, resultado de actividades antrópicas tiene un impacto negativo en la distribución espacial de las comunidades bióticas, modificando su estructura. Los macroinvertebrados bentónicos, son organismos acuáticos visibles a simple vista que viven enterrados en el lodo, o debajo de piedras y hojarascas; estos organismos responden frente a gradientes de contaminación orgánica, principalmente, origina cambios en la estructura de la comunidad.

Los índices bióticos y físico químicos son los más adecuados para evaluar las características ambientales en las aguas [2].

Los macroinvertebrados acuáticos son organismos que responden a tensores de contaminación de acuerdo a mecanismos de tolerancia, las especies euritípicas de mayor rango de tolerancia conquistan un mayor espacio geográfico, y las especies estenotípicas, menos adaptadas a cambios de las variables abióticas, tienden a desaparecer y otras especies no experimentan ninguna variación.

Muchos de los ecosistemas tropicales experimentan cambios biológicos considerables, dependientes principalmente de las épocas de sequía (verano) y lluvias (invierno). Como es natural, el estudio de un ecosistema con estas características debe llevarse a cabo en el espacio, y en el tiempo, para así identificar y comprender sus procesos de cambio [3].

El registro de los factores abióticos y bióticos de un ecosistema acuático permite interpretar la estructura y función del cuerpo de agua, que es de trascendencia para la calidad de vida de la población [4].

El Grupo de investigaciones ambientales ha desarrollado diversos estudios en el Río Pamplonita que incorporan la toma de datos biológicos y físico químicos para su posterior evaluación de la calidad ecológica. Entre otros, desde el año 2001 hasta el año 2012 se han desarrollado los siguientes: macroinvertebrados acuáticos como indicadores de la calidad del agua de la zona media del río Pamplonita; Estudio limnológico de la zona alta del río Pamplonita; estudio limnológico de la zona baja y el diseño del plan de monitoreo de la calidad y cantidad del recurso hídrico de las cuencas de los Ríos Algodonal, Zulia, Pamplonita y la subcuenca del río Táchira del Departamento Norte de Santander [5], [6].

Los cambios se pueden evidenciar por la presencia o ausencia de ciertos organismos, $\mathrm{o}$ mediante un análisis ecológico que incorpore variables bióticas y abióticas. El objetivo del proyecto es determinar los cambios espacio temporales de las variables bióticas y abióticas 
que afectan la dinámica de la comunidad de macroinvertebrados acuáticos presentes en el Río Pamplonita, en la parte alta y media, con el fin de establecer una base de conocimiento para el seguimiento y monitoreo del estado ecológico del río.

Un segundo objetivo propuesto es realizar la validación del modelode evaluación ecológica, el cual conjuga el comportamiento de la comunidad biótica y la respuesta ambiental ante diversos gradientes de contaminación. Los resultados de la variable ICOMO correlacionados con los datos de composición y estructura de los macroinvertebrados acuáticos fueron datos de entrada para modelar y simular la dinámica ecológica del río Pamplonita en una proyección de diez años [7].

\section{Materiales y métodos}

\subsection{Materiales}

Los materiales utilizados para su estudio fueron:

- Estereoscopio Wild M3

- Red surber de 25 x $25 \mathrm{~cm}$ y 500 micras

- Red D-Net

- Vasijas blancas

- Envases plásticos

\subsection{Métodos}

El estudio se desarrolló en cinco fases planteadas en función del cumplimiento de los objetivos del proyecto. Para ello se establecieron 6 estaciones de monitoreo distribuidas en la parte alta y media del río Pamplonita. El muestreo se realizó con una frecuencia de 1 muestra por mes, durante 7 meses, para un total de 42 muestras distribuidas durante los tiempos bimodales de lluvia y sequía.
2.2.1 Fase 1. Recopilación de información secundaria. La primera fase consistió en la consolidación de una base de conocimiento. En esta etapa, se realizó la revisión en diferentes fuentes bibliográficas de teorías, conceptos, antecedentes de proyectos limnológicos realizados en sistemas acuáticos lóticos a nivel nacional, regional y local. El producto de esta etapa contextualiza el estado del arte del tema objeto de estudio, normatividad vigente de calidad de aguas, textos guía de claves taxonómicas para identificación de las morfofamilias, artículos y documentos referentes de comparación y análisis.

2.2.2 Fase 2. Trabajo de campo. La segunda fase consistió en el diseño y desarrollo del trabajo de campo para la toma de datos in situ de macroinvertebrados acuáticos y parámetros físico químicos. La planeación para la captura de organismos responde al resultado de la aplicación de herramientas estadísticas como fue la ecuación de población desconocida para determinar el número de réplicas en la toma de macroinvertebrados acuáticos en cada estación de monitoreo. Acorde a la normatividad vigente, se solicitó el permiso ante CORPONOR para recolección de especímenes de la fauna acuática, con fines de estudios ambientales.

\section{Localización de las estaciones de monitoreo.}

La ubicación de las estaciones de muestreo se realizó a partir de criterios técnicos, características físicas del lecho y caudal del río, así como sitios definidos por estudios previos. Las estaciones fueron distribuidas espacialmente en función del gradiente altitudinal sobre la cuenca del Río Pamplonita. Se ubicaron tres en la parte alta (nacimiento del río hasta los 1000 m.s.n.m.), y tres en la zona media (1000-200 m.s.n.m.). En la tabla I, se presentan los datos de coordenadas y características generales de cada una de las seis estaciones de muestreo.
Enero - Junio 2017 ISSN 0122-820X E-ISSN 2422-5053 PP: 112-128

\section{5}


Diagnóstico espacio temporal de las variables abióticas y bióticas, que afectan la estructura de la comunidad de los macroinvertebrados bentónicos, presentes en un ecosistema lótico

Sistema de coordenadas: MAGNA Colombia Bogotá.

Datum: MAGNA

Falso Este: 1000000

Falso Norte: 1000000
Meridiano central: $-74,0775$

Factor de escala: 1,0000

Origen de la latitud: 4,5962

Unidades: metros

Tabla I. Localización de las estaciones de monitoreo

\begin{tabular}{|c|c|c|c|c|c|}
\hline Estación & $\begin{array}{l}\text { Nombre } \\
\text { estación }\end{array}$ & $\begin{array}{c}\text { Coordenada } \\
\text { (X) }\end{array}$ & $\begin{array}{l}\text { Coordenada } \\
\text { (Y) }\end{array}$ & $\begin{array}{c}\text { Altura } \\
\text { (m.s.n.m) }\end{array}$ & Características ambientales \\
\hline 1 & $\begin{array}{c}\text { La } \\
\text { Esperanza }\end{array}$ & 1.157 .375 & 1.307 .017 & 2316 & $\begin{array}{l}\text { Zona urbana de Pamplona, } 10 \text { metros aguas arriba se } \\
\text { evidencia descarga de aguas negras. El lecho del río } \\
\text { pedregoso y su cauce delimitado por un canal. }\end{array}$ \\
\hline 2 & $\begin{array}{l}\text { Terminal } \\
\text { Pamplona }\end{array}$ & 1.158 .421 & 1.307 .605 & 2295 & $\begin{array}{l}\text { Zona urbana de Pamplona, descargas puntuales de aguas } \\
\text { residuales. Lecho del río lodoso en su mayor porcentaje. }\end{array}$ \\
\hline 3 & $\begin{array}{l}\text { Villa } \\
\text { Marina }\end{array}$ & 1.159 .018 & 1.325 .060 & 1155 & $\begin{array}{c}\text { En este punto se observa un caudal abundante con fuerte } \\
\text { velocidad. Lecho pedregoso y arenoso, alta concentración } \\
\text { de sedimentos de origen mineral., no se observan } \\
\text { descargas puntuales sobre el río. }\end{array}$ \\
\hline 4 & Bocatoma & 1.168 .665 & 1.348 .420 & 523 & $\begin{array}{c}\text { En inmediaciones de la estación de muestreo se observa la } \\
\text { infraestructura de un punto de control de Ecopetrol, y paso } \\
\text { de volquetas que sustraen material del río. Aguas rápidas } \\
\text { con escasa presencia de actividad biológica. Lecho del } \\
\text { río arenoso. }\end{array}$ \\
\hline 5 & San Rafael & 1.173 .867 & 1.362 .168 & 311 & $\begin{array}{l}\text { Presencia de bosque ripario propio de bosque seco } \\
\text { tropical, sin embargo aguas arriba del punto de muestreo } \\
\text { existen descargas de aguas negras, disposición de residuos } \\
\text { sólidos sobre el río. }\end{array}$ \\
\hline 6 & Cerrito & 1.176 .198 & 1.371 .639 & 263 & $\begin{array}{l}\text { El punto de muestreo ya ha recibido las descargas de aguas } \\
\text { residuales del área urbana de Cúcuta, lecho lodoso, y una } \\
\text { mayor profundidad por aumento del volumen del agua } \\
\text { debido al vertimiento del caudal de aguas negras. }\end{array}$ \\
\hline
\end{tabular}

Fuente: autores

Muestreo de variables físico químicas. El muestreo in situ para muestras puntuales de las variables físico químicas se realizó en cada una de las 6 estaciones. Los parámetros medidos en campo fueron la temperatura ambiental y del agua, conductividad, $\mathrm{pH}$, dureza y sólidos disueltos totales. Adicionalmente, se realizó el análisis del oxígeno disuelto con el método Winkler.

Se procedió a la toma de muestras de un litro de agua siguiendo la metodología establecida en la Guía para el monitoreo de vertimientos, aguas superficiales y subterráneas [8]. Los envases fueron rotulados con la respectiva custodia para su transporte al laboratorio de la Universidad Francisco de Paula Santander. A estas variables se les mide generalmente su concentración, es decir la cantidad del elemento o compuesto presente en un determinado volumen de agua. Las variables procesadas en laboratorio fueron: demanda dioquímica de oxígeno $\left(\mathrm{DBO}_{5}\right)$, alcalinidad y acidez. Para el análisis de coliformes totales en unidades de número más probable (NMP) se recolectó la muestra de agua en un recipiente de vidrio esterilizado con capacidad de 0,5 Litros.

Muestreo para la determinación de las variables bióticas. La comunidad de macroinvertebrados acuáticos que ocupan distintos hábitats en el ecosistema, está conformada por individuos que se encuentran enterrados en el lecho del río, debajo de piedras, asociados a raíces de plantas y de hojas en descomposición.

Para la colecta y análisis cuantitativo se empleó una red surber de 25 x $25 \mathrm{~cm}$ y 500 micras de ojo de malla; se capturaron los macroinvertebrados asociados a hábitats bentónicos. Los organismos colectados en la red se separaron de acuerdo a las características morfológicas y se disponen en envases plásticos, los cuales contenían solución FAGA 1:1 (formol, alcohol, glicerina y agua) 
para su preservación. Los frascos se rotularon debidamente con la custodia. ¡Error! No se encuentra el origen de la referencia.

La colecta de macroinvertebrados bentónicos se realizó en un área total de $1 \mathrm{~m}^{2}$ en cada estación de monitoreo. Se realizaron 5 réplicas por cada sitio de muestreo y por cada muestreo [3].

\subsubsection{Fase 3. Análisis de laboratorio}

Análisis físico químico. Para la determinación de las variables abióticas de $\mathrm{DBO}_{5}$, dureza, alcalinidad, acidez y coliformes totales se siguieron las guías de laboratorio de Biotecnología Ambiental [9] de la Universidad Francisco de Paula Santander.

\section{Determinación de las familias de macroinvertebrados bentónicos. Las} muestras biológicas fueron depositados en cajas de petri, para ser observados a través del estereoscopio Wild M3, oculares 25X y 10X, objetivos 6, 4, 16 y 40, iluminación: fuente de luz de fibra óptica[4].

\subsubsection{Fase 4. Tratamiento de datos y análisis de la información}

Tratamiento de datos físico químicos. Los resultados se organizaron en matrices de acuerdo a los resultados espacio temporales, es decir por estaciones y fechas de monitoreo. Para obtener el Índice de Contaminación por materia orgánica ICOMO, los datos de Oxígeno disuelto en $\mathrm{mg} / \mathrm{L}$, se convierten en \% de saturación de Oxígeno Disuelto, utilizando la tabla de conversión [10] ingresando los valores de Oxígeno disuelto en ppm, altitud en m.s.n.m. y los valores de temperatura del agua. El principio fundamental de realizar la conversión, es la afectación de la presión y la temperatura sobre la cantidad de oxígeno real presente en el agua, y disponible para las funciones vitales de los organismos acuáticos. Posteriormente se utilizó el software
ICATEST V1.0 [11], por medio del cual se realizó el análisis comparativo a partir de la aplicación de los índices de contaminación propuestos en [12], como son ICOMO (Índice de contaminación por materia orgánica), ICOSUS (Índice de contaminación por sólidos totales), ICOMI (índice de contaminación por mineralización), ICOpH (índice de contaminación por $\mathrm{pH})$.

El Análisis Multivariado de Componentes Principales (ACP) se realizó en concordancia con las recomendaciones explícitas en [13], a partir del establecimiento de una matriz de monitoreo (estaciones-periodos) Vs. Variables.

Igualmente se aplicaron técnicas de estadística descriptiva a los resultados de los índices de contaminación por materia orgánica, para generar los mínimos y máximos en cada estación por fecha de muestreo.

Tratamiento de datos Biológicos. Utilizando diferentes claves taxonómicas, se organizaron los macroinvertebrados acuáticos a nivel de familia, posteriormente se determinaron los resultados de diversidad por estación y por muestreo.

Para los análisis de estructura de la población bentónica se analizaron los indicadores de densidad, organismos presentes en un espacio dado, proporción y abundancia. Índices de diversidad de Shannon-Wiener. Margalef y Menhinik. Se determinó la disimilitud de Bray Curtis entre estaciones. La aplicación de estos ndices se realizó utilizando las herramientas computacionales de Biodiversity Pro y Past.

Índices de diversidad en (1), (2), (3) y (4):

Shannon-Wiever (1949):

$$
H^{\prime}=-\sum_{i=1}^{S} \frac{n i}{n} \operatorname{Ln} \frac{n i}{n}
$$


Margalef: $\quad d=\frac{s-1}{L n n}$

Menhinick: $d=\frac{s}{\sqrt{n}}$

Disimilitud de Bray Curtis (1957).

$$
D_{j k}=\frac{\sum_{j=1}^{S}\left\lfloor X_{i j}-X_{i k}\right\rfloor}{\sum_{j=1}^{S}\left\lfloor X_{i j}-X_{i k}\right\rfloor}
$$

FASE 5. Validación del modelo: Modelación dinámica de una comunidad de macroinvertebrados bentónicos frente a un gradiente temporal de contaminación orgánica.

A partir de los rangos de tolerancia de cada familia frente al ICOMO definido con los datos de abundancia de las morfofamilias presentes en cada periodo y estación de monitoreo, en función de los valores máximos, medio y mínimos del índice de contaminación por materia orgánica, se aplicó y validó la propuesta metodológica: "modelación dinámica de una comunidad de macroinvertebrados bentónicos frente a un gradiente temporal de contaminación orgánica" a partir de los rangos de tolerancia de cada organismo encontrado frente al ICOMO [14].

El Índice de Contaminación por Materia Orgánica ICOMO de acuerdo a los planteamientos definidos en [15], está conformado por las variables: Demanda Bioquímica de Oxígeno $\left(\mathrm{DBO}_{5}\right)$, Coliformes Totales y Porcentaje de Saturación de Oxígeno, que son incorporadas en (5)

$$
\begin{gathered}
I C O M O=\frac{1}{3} I \cdot D B O \\
\text { Donde }: I_{\cdot D B O 5}=-0.05+0.70 \log _{10}+D B O\left(\frac{\mathrm{mg}}{\mathrm{L}}\right) \\
\mathrm{DBO}>30(\mathrm{mg} / \mathrm{l})=1, \quad \mathrm{y} \quad \mathrm{DBO}<2(\mathrm{mg} / \mathrm{l})=0 . \\
I_{\cdot C \text { Coliformes Totales }}=-1.44+0.56 \log _{10} \text { Col Totales }\left(\frac{\mathrm{NPM}}{100 \mathrm{ml}}\right) \\
\text { C. Totales }>20.000(\mathrm{NMP} / 100 \mathrm{ml})=1, \text { y C. Totales }<500(\mathrm{NMP} / 100 \mathrm{ml})=0 . \\
I \cdot \text { oxígeno Disuelto } \%=1-0.01 \text { Oxígeno } \%
\end{gathered}
$$

Oxígenos (\%) mayores a 100\% tienen un índice de oxígeno de 0.

Con los rangos de tolerancia se establecieron los índices para cada una de las morfofamilias de macroinvertebrados acuáticos en función del ICOMO, que representen variabilidad y espacio temporal de la dinámica de los organismos presentes en el Río Pamplonita.

Para el diseño del modelo se utilizaron diagramas de Forrester y mediante el uso del Software Stella V. 8.1 se introducen las ecuaciones matemáticas de la propuesta metodológica, incorporando los resultados de
ICOMO, funciones de índices de tolerancia y abundancias de macroinvertebrados acuáticos; los datos simularon escenarios del comportamiento dinámico y ecológico del sistema del Río Pamplonita estimado a 120 meses. Finalmente, se representó gráficamente la dinámica simulada de los macroinvertebrados bentónicos en el Río Pamplonita, y se obtuvo la generación de coenoclinas de abundancia de organismos en función de un gradiente de contaminación por materia orgánica. 


\section{Resultados y análisis}

\subsection{Resultados de variables físico químicas}

Se observó que el potencial de hidrógeno $(\mathrm{pH})$, en todas las estaciones de muestreo, excede el valor máximo establecido en la norma [16] que debe corresponder al valor de 9,0, mientras que los valores por debajo del mínimo de la norma igual a 6,5 se presentaron en la estación de La esperanza (Salida de campo 3 y 4), el terminal de Pamplona (salida de campo 1, 2, 3) y en la bocatoma (salida de campo 2). Se observó que el $88 \%$ de los resultados del muestreo, se encontraban por encima del valor máximo permitido. Comportamiento presentado en la publicación del índice biológico, BMWP (biological monitoring working party score), modificado y adaptado al Río Pamplonita [5].

La variación espacio temporal del parámetro de Oxígeno Disuelto (mg/L), registró valores por debajo de la norma, llegando a niveles de $0 \mathrm{mg} / \mathrm{L}$; este fenómeno se observó en las estaciones 2 y 6 , estaciones localizadas aguas abajo de la descarga de aguas residuales de zonas urbanas; como respuesta al incremento de la materia orgánica, en estos puntos, se registra un alto porcentaje de abundancia de macroinvertebrados bentónicos de carácter euritípico, muy tolerantes a este tipo de contaminación; las familias más abundantes en estas estaciones, fueron Psychodidae con un $75 \%$ en la estación 2 , y con el $25 \%$ de abundancia en la estación 6 , seguida de la familia Tubificidae, con un $37,5 \%$ en la estación 2 , y el $25 \%$ en la estación 6 . Los resultados son semejantes al estudio realizado en el año 2002, en la estación 6, del cerrito, respecto al reporte de las familias como a la abundancia..

Los demás datos tomados en las diferentes estaciones se encontraron sobre el valor establecido en la norma, sin embargo, la presencia de coliformes en todos los sitos de muestreo, establece que el agua no es apta para el consumo humano, para el desarrollo de actividades agrícolas ni para recreación por el riesgo de patógenos presentes en el cuerpo de agua.

La temperatura del agua tomada en las estaciones 1 y 2 , osciló entre 14 y $18{ }^{\circ} \mathrm{C}$; en las estaciones $3,4,5$ y 6 , la temperatura permaneció en un rango entre 24 y $26^{\circ} \mathrm{C}$. Por lo tanto, puede afirmarse que esta variable presentó una relación dependiente de la variación espacial (gradiente altitudinal).

Laestación6, evidenció unaalta conductividad, debido al proceso de mineralización y la presencia de material de arrastre con elevada concentración de sedimentos en el agua. En esta estación las familias más frecuentes fueron Chironomidae y Glossiphoniidae.

La dureza presentó valores por encima de la norma [17], en todas las estaciones durante el muestreo del mes de mayo, periodo de sequía. La estación con mayor dureza fue la estación 6 , esta variable está asociada con la alcalinidad, conductividad y $\mathrm{pH}$ alto.

La demanda bioquímica de oxígeno presentó un comportamiento con valores altos en todas las estaciones, principalmente en la estación 2 igual a $128 \mathrm{mg} / \mathrm{L}$, el valor promedio de este parámetro fue de $28,3 \mathrm{mg} / \mathrm{L}$. Lo anterior, se ve reflejado por las familias más abundantes de los macroinvertebrados bentónicos encontrados, que corresponde a organismos tolerantes y muy tolerantes a la presencia de materia orgánica.

Enero - Junio 2017

SN 0122-820X

$112-128$

En la tabla II, se observan los resultados de estadística descriptiva aplicada a los parámetros físicos y químicos, el índice de Contaminación por Materia Orgánica ICOMO, índice de Contaminación por Mineralización ICOMI, y el índice de Contaminación por pH $\mathrm{ICOpH}$. 
Diagnóstico espacio temporal de las variables abióticas y bióticas, que afectan la estructura de la comunidad de los macroinvertebrados bentónicos, presentes en un ecosistema lótico

Tabla II. Estadística descriptiva de las variables físico químicas

\begin{tabular}{|c|c|c|c|c|c|}
\hline PARÁMETRO & PROMEDIO & MÍNIMO & MÁXIMO & MEDIANA & MODA \\
\hline Potencial de Hidrógeno $(\mathrm{pH})$ & 7,94 & 4,87 & 11,53 & 7,86 & 8 \\
\hline Temperatura Ambiente $\left({ }^{\circ} \mathrm{C}\right)$ & 25,35 & 18,3 & 31,5 & 25,8 & 22 \\
\hline Temperatura Agua $\left({ }^{\circ} \mathrm{C}\right)$ & 21,22 & 14,5 & 26,7 & 22,1 & 24 \\
\hline Oxígeno Disuelto (OD) $\mathrm{mg} / \mathrm{L}$ & 6,8 & 2,9 & 11 & 7,2 & 58 \\
\hline \% Saturación OD & 68 & 32 & 108 & 62,5 & 58 \\
\hline Conductividad (mS) & 0,19 & 0,03 & 0,45 & 0,18 & 0,18 \\
\hline Sólidos Totales Disueltos (ppt) & 0,16 & 0,03 & 0,52 & 0,12 & 0,09 \\
\hline Acidez (mg/L) & 8,85 & 3,4 & 29 & 7 & 6 \\
\hline Alcalinidad (mg/L) & 17,24 & 4 & 105 & 9 & 9 \\
\hline Dureza Total (mg/L) & 158,83 & 30 & 370 & 145 & 110 \\
\hline Dureza cálcica $(\mathrm{mg} / \mathrm{L})$ & 98,52 & 30 & 250 & 90 & 90 \\
\hline Dureza del magnesio $(\mathrm{mg} / \mathrm{L})$ & 60,31 & 10 & 156 & 50 & 30 \\
\hline Demanda Bioquímica de Oxigeno DBO5 (mg/L) & 37,11 & 1,95 & 128 & 28,3 & 26 \\
\hline ICOMO & 0,63 & 0,17 & 0,89 & 0,65 & 0,62 \\
\hline ICOMI & 0,47 & 0,09 & 0,78 & 0,48 & 0,67 \\
\hline $\mathrm{ICOpH}$ & 0,18 & 0 & 1 & 0,019 & 0 \\
\hline
\end{tabular}

Fuente: autor

Se encontró que el valor promedio de ICOMO fue de 0,63 . El valor máximo igual a 0,89 y el valor mínimo de 0,17 . El valor que más se repite es de 0,62 de aguas contaminadas por materia orgánica. Este comportamiento se observó en todas las estaciones de monitoreo. los componentes 1 y 2 , representan el comportamiento del $84 \%$ de varianza. Los valores se encuentran en rangos de aguas contaminadas a muy contaminadas, esta situación se evidenció en las seis estaciones durante los siete monitoreos.

El análisis de componentes principales se observa en la Figura1. Los valores de

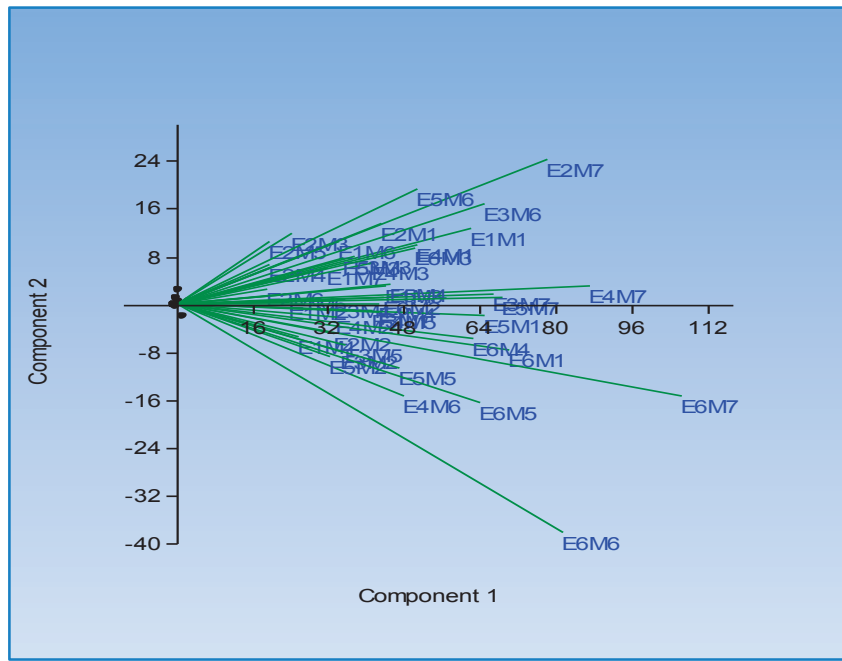

Figura. 1. Biplot de variables físico químicas

Fuente: Autores

\subsection{Resultados biológicos}

Se registraron un total de 115 familias en los 42 muestreos realizados en la parte alta y media del río Pamplonita. Sin embargo, para la validación del modelo se realizó un proceso de selección teniendo como criterio técnico la frecuencia de aparición del organismo, de por lo menos 3 veces del registrado durante todo el monitoreo. Una vez realizado dicho 
proceso, se determinaron un total de 12 familias. Como se muestra en la tabla III las condiciones de carga contaminante durante todos los muestreos y estaciones, fue el mayor tensor ambiental que afectó la presencia de 103 organismos euritípicos a la materia orgánica.

Tabla III. Familias de macroinvertebrados acuáticos presentes en la parte alta y media del río pamplonita.

\begin{tabular}{|c|c|}
\hline FAMILIA & NUMERO DE INDIVIDUOS \\
\hline Hyalellidae & 4 \\
\hline Dugesidae & 37 \\
\hline Chordodidae & 7 \\
\hline Lumbriculiade & 2 \\
\hline Tubificidae & 8 \\
\hline Glossiphoniidae & 62 \\
\hline Baetidae & 122 \\
\hline Tricorythidae & 49 \\
\hline Leptophlebiidae & 33 \\
\hline Psychodidae & 4 \\
\hline Chironomidae & 38 \\
\hline Dytiscidae & 4 \\
\hline TOTAL & $\mathbf{3 7 0}$ \\
\hline
\end{tabular}

Fuente: Autores

Las familias más frecuentes en su orden fueron la familia Baetidae con un total de 122 individuos encontrados, es decir el $33 \%$ de abundancia; la familia Glossiphoniidae con un total de 62 organismos, equivalente al 16,8 $\%$ de abundancia; la familia Tricorythidae con un total de 49 individuos, finalmente la familia Chironomidae con un total de 38 organismos. Estas familias presentaron un comportamiento tolerante a la contaminación por materia orgánica. El total de individuos registrados de las doce familias seleccionadas fue de 370 .

La tabla IV, presenta los resultados de frecuencia en monitoreos, abundancia, proporción y densidad de individuos por $\mathrm{m}^{2}$ de área de muestreo.

Tabla IV. Abundancia, proporción y densidad de las familias más frecuentes de macroinvertebrados acuáticos reportados en el Río Pamplonita, durante el monitoreo de las 6 estaciones.

\begin{tabular}{|c|c|c|c|c|}
\hline Familia & $\begin{array}{c}\text { Total de } \\
\text { individuos }\end{array}$ & $\begin{array}{c}\text { Frecuencia en } \\
\text { monitoreo }\end{array}$ & $\begin{array}{c}\text { Abundancia } \\
(\mathbf{\%})\end{array}$ & $\begin{array}{c}\text { Dens. o Abun. Ind * } \\
\text { m2 }\end{array}$ \\
\hline Hyalellidae & 4 & 3 & 1,1 & 1,3 \\
\hline Dugesidae & 37 & 12 & 10,0 & 3,1 \\
\hline Chordodidae & 7 & 4 & 1,9 & 1,8 \\
\hline Lumbriculiade & 2 & 2 & 0,5 & 1 \\
\hline Tubificidae & 8 & 4 & 2,2 & 2,0 \\
\hline Glossiphoniidae & 62 & 18 & 16,8 & 3,4 \\
\hline Baetidae & 122 & 17 & 33,0 & 7,2 \\
\hline Tricorythidae & 49 & 13 & 13,2 & 3,8 \\
\hline Leptophlebiidae & 33 & 4 & 8,9 & 8,3 \\
\hline Psychodidae & 4 & 2 & 1,1 & 2,0 \\
\hline Chironomidae & 38 & 4 & 10,3 & 9,5 \\
\hline Dytiscidae & 4 & 3 & 1,1 & 1,3 \\
\hline Total & 370 & & 100,00 & \\
\hline
\end{tabular}

Fuente: Autores

La variable densidad se calculó del total de individuos y la frecuencia en los monitoreos, por el área de muestreo de $1 \mathrm{~m}^{2}$. La familia de mayor densidad fue la familia Chironomidae con un valor igual a 9,5 .
Los resultados de diversidad de Shannon presentaron un promedio igual a 0,62 nits/Ind de condición de diversidad muy baja. Según los registros de la tabla $\mathrm{V}$, el menor valor de diversidad se presentó en la estación 4 , con un índice igual a 0,3 de condición de diversidad 
Shannon de muy baja, lo mismo se presentó en la diversidad de Margalef y Menhinick de condición de diversidad alfa muy baja.

En el estudio realizado en el año 2001, la diversidad de esta estación fue de 1,7 en [4].

Se evidenció en la zona 4, una gran afectación de las condiciones naturales del río, como el lecho arenoso, la cantidad de material pétreo del río ha disminuido, afectando la composición y estructura de las comunidades bentónicas. Adicionalmente, como es un paso de volquetas para el trasporte de material pétreo, esto ha ocasionado un impacto negativo que afecta drásticamente las condiciones de diversidad del sistema lótico.

El promedio valor de diversidad de Shannon se observó en la estación 5, igual a 0,85 nits/ Ind, considerándose un valor muy bajo.

Tabla V. índices de diversidad

\begin{tabular}{|c|c|c|c|c|c|c|}
\hline INDICE & E1 & E2 & E3 & E4 & E5 & E6 \\
\hline Taxa_S & 3,67 & 1,67 & 2,29 & 1,71 & 3 & 2,43 \\
\hline Individuals & 8,33 & 4,33 & 9,57 & $\mathbf{1 4}$ & 12,8 & 12,29 \\
\hline Shannon_H & 0,82 & 0,4 & 0,67 & $\mathbf{0 , 3}$ & $\mathbf{0 , 8 5}$ & 0,67 \\
\hline Menhinick & 1,04 & 0,81 & 0,74 & $\mathbf{0 , 4 7}$ & 0,85 & 0,74 \\
\hline Margalef & 1,24 & 0,42 & 0,55 & $\mathbf{0 , 2 7}$ & 0,70 & 0,59 \\
\hline
\end{tabular}

Un total de 12 familias de macroinvertebrados acuáticos prefieren hábitats con presencia de materia orgánica. De acuerdo a los resultados obtenidos de estructura de la comunidad, la mayor diversidad se encontró en la estación 2 , durante el primer muestreo, el cual registra la mayor diversidad de Shannon igual a 2,01 de condición es de diversidad media; esta situación se presentó debido a la presencia de organismos muy tolerantes a la contaminación. Se registraron durante todos los 42 muestreos rangos bajos a muy bajos de diversidad.

La estación 4 presentó un mayor número de individuos el $26,5 \%$, pero menor número de familias, y un valor de promedio de ICOMO igual a 0,6 de contaminación media; mientras que la estación con menor número de individuos fue la estación 1, con un porcentaje igual al 6,76\%, y un valor de ICOMO de 0,79 de contaminación muy alta (Figura 2).

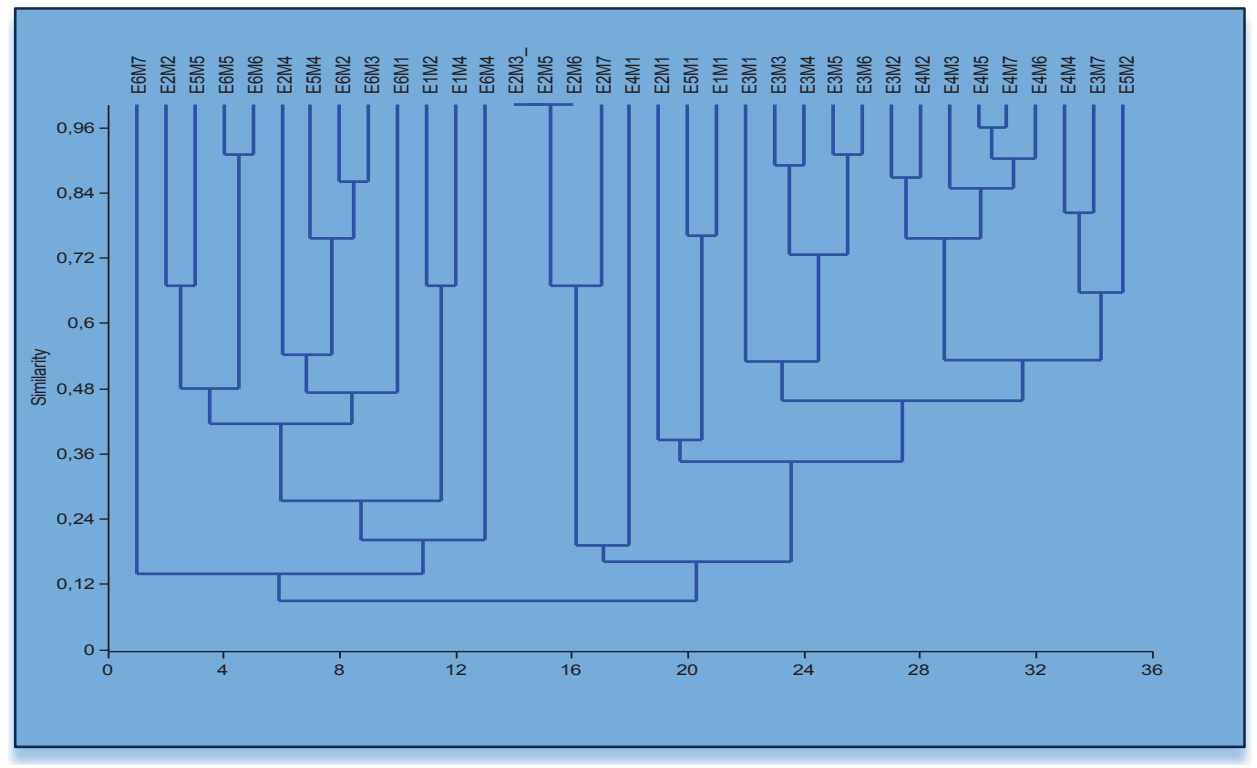

Figura 2.Disimilitud de Bray Curtis entre estaciones Fuente: Autores 
Los resultados de disimilitud entre las estaciones, mostraron que las estaciones de Villa Marina y Bocatoma son las de mayor similitud con un $84 \%$. Mientras que, con el $15 \%$ de menor similitud se encontraron las estaciones de la Bocatoma y la del terminal de Pamplona.

\subsection{Validación del modelo}

La validación del modelo se realizó a partir de los monitoreos desarrollados en el río Pamplonita, en su parte alta y media, teniendo en cuenta los requerimientos de información de acuerdo al modelo propuesto. De esta manera, la selección de taxas se realizó a partir de la representatividad del porcentaje de abundancia en el total de individuos de la comunidad de los macroinvertebrados acuáticos encontrados. Teniendo en cuenta lo anterior, se definen 12 taxas como insumo. Los rangos de máximo y mínimo de tolerancia para cada una de las taxas se presenta en la tabla VI; los valores registrados oscilan entre el valor mínimo de ICOMO igual a 0,2 , y el máximo valor igual a 0,9 .

Dentro de la clasificación de la tolerancia a la CO (Contaminación Orgánica), los resultados fueron organismos clasificados como tolerantes a muy tolerantes, sin embargo, es importante resaltar que se observan diferencias en cuanto a la amplitud del rango del Índice de Contaminación por materia orgánica (ICOMO) con respecto al modelo metodológico propuesto. De esta manera, la determinación de la categoría de la tolerancia a la $\mathrm{CO}$ de los organismos encontrados, se realizó con base en la columna de rango de índices de tolerancia de la tabla VI.

Tabla VI. Rango de índice de tolerancia al icomo

\begin{tabular}{|c|c|c|c|}
\hline Familia & Mínimo & Medio & Máximo \\
\hline Hyalellidae & 0,7 & 0,8 & 0,9 \\
\hline Dugesidae & 0,3 & 0,5 & 0,7 \\
\hline Chordodidae & 0,5 & 0,6 & 0,8 \\
\hline Lumbriculiade & 0,7 & 0,8 & 0,9 \\
\hline Tubificidae & 0,5 & 0,7 & 1,0 \\
\hline Glossiphonidae & 0,4 & 0,6 & 0,9 \\
\hline Baetidae & 0,2 & 0,5 & 0,9 \\
\hline Tricorythidae & 0,2 & 0,5 & 0,9 \\
\hline Leptophlebiidae & 0,5 & 0,6 & 0,7 \\
\hline Psychodidae & 0,7 & 0,8 & 1,0 \\
\hline Chironomidae & 0,7 & 0,8 & 0,9 \\
\hline Dytiscidae & 0,6 & 0,7 & 0,8 \\
\hline
\end{tabular}

Fuente: Autores

La definición de las funciones o índices de tolerancia (IT) para las 12 familias, se realizó a partir de la tabla IV, que relaciona el patrón de abundancia de los MIA como respuesta al gradiente de ICOMO. A partir de esta relación se establece la tabulación de los IT definidos en la Tabla VI. Nótese que los valores oscilan entre los rangos mínimo, promedio y máximo de tolerancia, lo que permite observar y simular la respuesta de las abundancias de los taxa y con ello de la diversidad de la comunidad de macroinvertebrados bentónicos en su conjunto, ante el efecto de tensores de contaminación orgánica, bien sean éstos de carácter espacial o temporal.

La tabla VII, consolida los insumos de información para las 12 familias, a partir de los rangos de ICOMO, y los patrones de abundancia. Se define la categoría de tolerancia, y la categoría de ICOMO. 
Diagnóstico espacio temporal de las variables abióticas y bióticas, que afectan la estructura de la comunidad de los macroinvertebrados bentónicos, presentes en un ecosistema lótico

Tabla VII. Datos de macroinvertebrados acuáticos insumos para la validación del modelo

\begin{tabular}{|c|c|c|c|c|c|c|}
\hline Familia & $\begin{array}{l}\text { Rango } \\
\text { ICOMO }\end{array}$ & $\begin{array}{c}\text { Categoría de tolerancia } \\
\text { a la Co }\end{array}$ & $\begin{array}{c}\text { Categoría } \\
\text { ICOMO }\end{array}$ & $\begin{array}{c}\text { Frecuencia en } \\
\text { monitoreos }\end{array}$ & Porcentaje & $\begin{array}{c}\text { Dens. o } \\
\text { Abun. Ind * } \\
\text { m2 }\end{array}$ \\
\hline Hyalellidae & $0,7-0,9$ & Muy tolerante & Muy alta & 3 & 1,08 & 1 \\
\hline Dugesiidae & $0,3-0,7$ & Tolerante & Alta & 12 & 10 & 3 \\
\hline Chordodidae & $0,5-0,8$ & Tolerante & Alta & 4 & 1,89 & 2 \\
\hline Lumbriculiade & $0,7-0,9$ & Muy tolerante & Muy alta & 2 & 0,54 & 1 \\
\hline Tubificidae & $0,5-1,0$ & Muy tolerante & Muy alta & 4 & 2,16 & 2 \\
\hline Glossiphonidae & $0,4-0,9$ & Muy tolerante & Muy alta & 18 & 16,76 & 3 \\
\hline Baetidae & $0,2-0,9$ & Muy tolerante & Muy alta & 17 & 32,97 & 7 \\
\hline Tricorythidae & $0,2-0,9$ & Muy tolerante & Muy alta & 13 & 13,24 & 4 \\
\hline Leptophlebiidae & $0,5-0,7$ & Tolerante & Alta & 4 & 8,92 & 8 \\
\hline Psychodidae & $0,7-1,0$ & Muy tolerante & Muy alta & 2 & 1,08 & 2 \\
\hline Chironomidae & $0,7-0,9$ & Muy tolerante & Muy alta & 4 & 10,27 & 10 \\
\hline Dytiscidae & $0,6-0,8$ & Tolerante & Alta & 3 & 1,08 & 1 \\
\hline
\end{tabular}

Fuente: Autores
Las familias más abundantes fueron Baetidae, Glossiphoniidae, Tricorythidae y Dugesiidae. La de menor abundancia fue la familia lumbriculiade. La familia Glossiphoniidae, de la clase Hirudinea, está conformada por organismos presentes en aguas lénticas eutróficas, y lóticas alfa, meso y oligosapróbicas. Solo unas pocas especies son hematófagas, se alimentan de residuos orgánicos carnívoras, consumen caracoles, insectos, lumbricidos, entre otros. Algunas parásitas de peces, ranas y otros en [5].

La estructura del modelo, se aprecia en la figura 3, así, en el nivel superior de acuerdo al modelo propuesto se halla la variable definida como ICOMO que representa el incremento de la $\mathrm{CO}$ en el tiempo que va de 0 a 1 . ICOMO sirve como entrada para cada una de los IT de los taxa y la relación particular ICOMO-ITi se construye a partir de los rangos de tolerancia individuales. A su vez, los IT sirvieron de entradas para el cálculo de las funciones de densidad (FD) de cada taxa expresadas en las variables Ch, Ba, Hyal, Glosi, Lept, Duge, Lumb, Dyt, Chor, Psyc, Tr y Tub incorporadas en las ecuaciones matemáticas del modelo. Los valores iniciales de cada taxa se toman a partir de la Tabla IV, resultado del análisis de abundancia, proporción y densidad de los individuos. La siguiente variable declarada en el modelo fue la densidad total de individuos (N) de la comunidad de MIB, establecida por la sumatoria de las densidades individuales. La razón entre las densidades de cada taxa y la total, constituyen a su vez los insumos para calcular el índice de diversidad de Shannon. La base de tiempo para todas las variables del modelo fue de 120 meses.

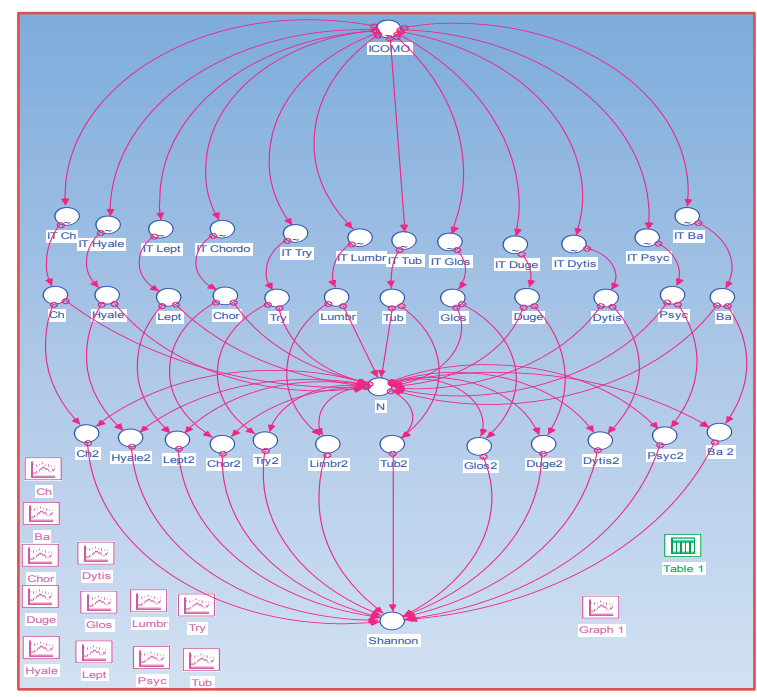

Figura 3. Diagrama de Forrester para la modelación del gradiente de contaminación orgánica. ICOMO: índice de contaminación orgánica;

Fuente: Autores

Con respecto a la dinámica de la diversidad de Shannon observada en los resultados de la simulación a 120 meses, en relación al incremento del ICOMO, en la figura 4, se observa que genera una curva Normal, que inicia su incremento a partir del mes 13 , ya que los 12 primeros meses cuando el ICOMO se encuentra en un rango de 0,0 a 0,10 la diversidad y el número de individuos es igual a 0 . Este fenómeno obedece a que todos los organismos encontrados en las seis estaciones requieren de materia orgánica para su crecimiento y desarrollo. La diversidad de Shannon llega a su máximo valor entre los meses 78 y 85 , cuando el ICOMO está en el 
rango de 0,65 y 0,72 , la diversidad alcanza su mayor valor de $\left(H^{\prime}=1,89\right)$, la Condición de Diversidad es Media [17]. Hasta el mes 85 este valor de $\left(\mathrm{H}^{\prime}\right)$ se vuelve constante, hasta el mes 86 donde inicia su decremento gradual y constante hasta el mes 120 que llega a $\left(\mathrm{H}^{\prime}=\right.$ 1,10) de una Condición de Diversidad Baja [17].

El incremento gradual del número de individuos de manera exponencial y paralelo al incremento del ICOMO se debe a que los organismos más abundantes en los datos insumo del modelo son de amplio rango de tolerancia a la C.O. Por lo tanto, en la medida que aumenta el ICOMO aumenta el número de individuos N. Sin embargo, a partir del mes 86 la función de $\mathrm{N}$ declina hasta llegar a valores de 3,72. Este fenómeno se presentó en la validación del modelo en los resultados del proyecto de macroinvertebrados acuáticos de la zona media del Río Pamplonita.

En la figura 5, se aprecia la dinámica de los MIB dentro de este grupo. Las taxas Hyalellidae y lumbriculiade, son las de mayor tolerancia a la CO, la familia Hyalellidae presenta su máxima densidad en el mes 108 con ICOMO de 0.9, la densidad decrece hasta el mes 120, cuando el ICOMO es igual a 1.0 , y su densidad es de 0.1. La familia Lumbriculiade presenta su mayor abundancia en el mes 107, cuando el ICOMO es igual a 0,89 . En términos generales los organismos presentan un índice de tolerancia entre los rangos tolerantes y muy tolerantes.

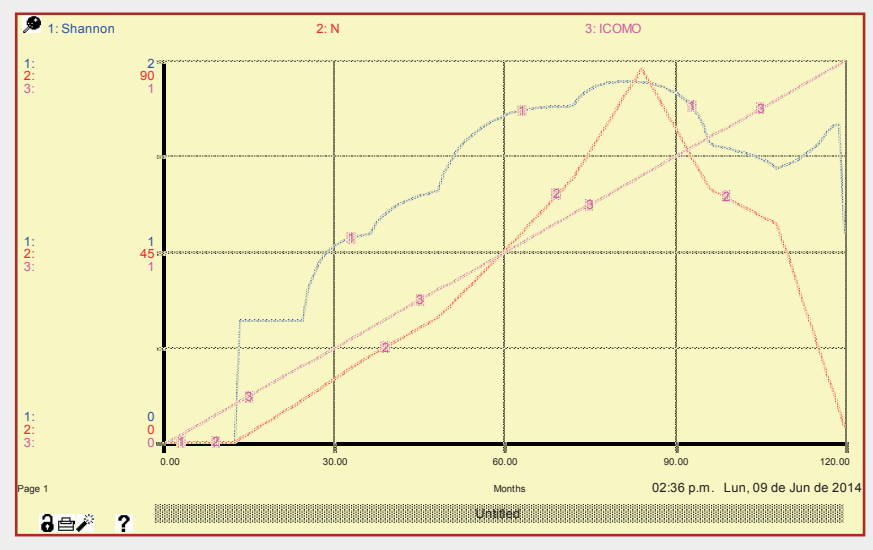

Figura 4. Dinámica temporal simulada de la diversidad (H: Shanon) y número total de individuos $(\mathrm{N})$ de la comunidad de mib, en contraste con el incremento de ICOMO, en un tiempo de 120 meses. Fuente: Autores

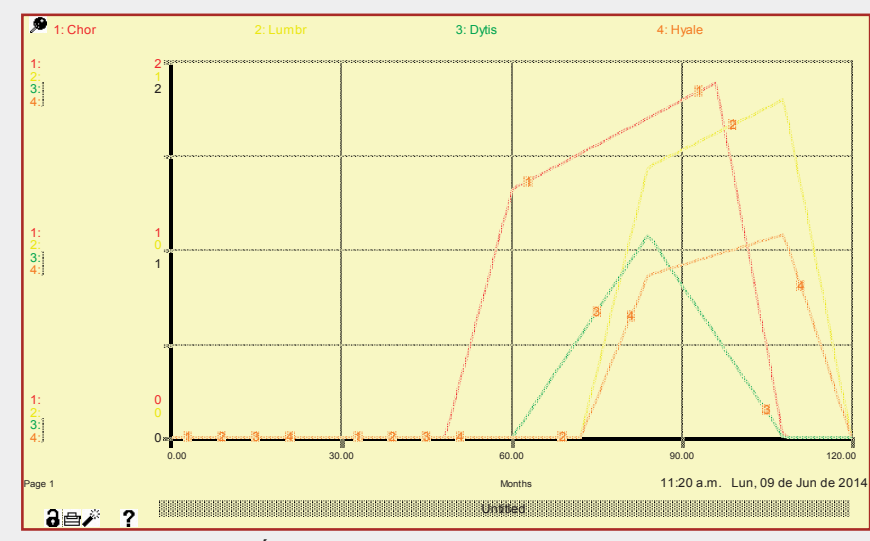

Figura 5. DINÁMICA TEMPORAL DE LA DENSIDAD DE INDIVIDUOS PARA LOS TAXA DE CHORDODIDAE, DYTISCIDAE, HYALELLIDAE Y LUMBRICULIADE.

Fuente: Autores 
Diagnóstico espacio temporal de las variables abióticas y bióticas, que afectan la estructura de la comunidad de los macroinvertebrados bentónicos, presentes en un ecosistema lótico

Se observó de acuerdo a los resultados de la simulación, que las familias Tubificidae y Psychodidae son las de mayor tolerancia a la materia orgánica; los Psychodidae son individuos aeropneústicos, es decir capturan oxígeno atmosférico para poder desarrollar sus funciones vitales.

La familia Chironomidae es muy tolerante a la C.O, mientras que Leptophlebiidae tiene un rango de mínimo de ICOMO igual a 0,4 en el mes 41 y un máximo de 0,8 en el mes 96 .

El modelo permitió obtener resultados de evaluación ecológica, incorporando variables bióticas y abióticas. De igual manera, permite estimar a 10 años el estado ecológico del sistema acuático frente a un gradiente de contaminación orgánica.

\section{Conclusiones}

Los resultados encontrados deben motivar la urgenciadeproyectosderestauraciónecológica del río, ya que en todas las estaciones desde la parte alta y la media del Río Pamplonita, se evidencia con análisis físico químicos y biológicos la afectación de la estructura de la comunidad de macroinvertebrados acuáticos.

Se identificaron un total de 115 familias de macroinvertebrados acuáticos presentes en las seis estaciones del Río Pamplonita, sin embargo de acuerdo a los insumos requeridos para el modelo ante la necesidad de generar valores máximos, medios y mínimos de tolerancia a la Contaminación por materia orgánica, solo se trabajó con 12 familias, que reportaron un comportamiento euritípico a la contaminación por materia orgánica. Las claves taxonómicas empleadas permitieron llegar al nivel de familia.

Los rangos de Tolerancia de las taxas encontradas oscilaron entre los valores de ICOMO de 0,2 como valor mínimo y 1,0 como máximo valor. Se encontraron que las familias más euritípicas a la contaminación por materia orgánica fueron las más abundantes en todos los muestreos.

Se validó la propuesta metodológica de modelación y simulación dinámica de la comunidad de macroinvertebrados bentónicos frente a un gradiente temporal de contaminación orgánica. Encontrándose que en comparación con los rangos definidos en otros sistemas acuáticos para las taxas de Baetidae y Tricorythidae, en el estudio realizado ampliaron su rango de tolerancia a la CO. Las familias Psychididae, Chironomidae y Tubificidae permanecen con su rango de muy tolerantes a la CO.

Los datos de la simulación del sistema del Río Pamplonita comprueban los planteamientos de Roldán (1998), que establece que a mayor contaminación menor diversidad y a menor contaminación mayor diversidad. Sin embargo, los macroinvertebrados acuáticos que han desarrollado mecanismos de tolerancia a altas concentraciones de materia orgánica aumentan en abundancia sobre las especies estenotipicas.

Las seis estaciones presentan contaminación y bajas condiciones de diversidad; cuatro de ellas: La Estación la Esperanza, Terminal de Pamplona, Puente San Rafael y El cerrito por descargas de aguas residuales de la población urbana, y en dos de ellas: Las estaciones Villa Marina y Bocatoma por la intervención de extracción de material de construcción eliminando por las poblaciones biológicas, aumentando las concentraciones de material particulado de tipo inorgánico que afecta la estructura de las comunidades de macroinvertebrados acuáticos (evidenciado en los muestreos y en los resultados de las variables bióticas y abióticas).

Las variaciones espaciales, determinadas por gradiente altitudinal, no son un factor determinante en la estructura de la comunidad 
de los macroinvertebrados encontrados en el estudio. La composición y estructura dependió en su mayor parte, de las condiciones de variación climática, descargas de aguas residuales, características del lecho del río y acciones antrópicas como extracción de material pétreo.

\section{Agradecimientos}

Los resultados del proyecto de investigación y el cumplimiento de los objetivos se lograron gracias a la participación activa de los integrantes del semillero de Ciencia y Tecnología ambiental del programa de Ingeniería de la Universidad Francisco de Paula Santander.

La financiación de las actividades de monitoreo del proyecto se realizó con recursos del FINU de la Vicerrectoría de Investigación y Extensión de la U.F.P.S.

\section{Referencias}

[1] G. Márquez, "Ecosistemas estratégicos de Colombia", julio 2003. [En línea]. Disponible: https://www.sogeocol.edu. co/documentos/07ecos.pdf.

[2] N. Prat, I. Muñoz, G. González, y X. Millet, "Comparación crítica de dos índices de calidad del agua: ISQ4 y Bill”, Tecnología del agua, vol. 31, pp. 33-49,1986.

[3] N. Fernández, A. Ramírez y F. Solano, "Dinámica físico química y calidad del agua en la Microcuenca el Volcán, Municipio de Pamplona, Colombia" Bistua, vol. 3, no.1, pp. 5-17, 2005.

[4] M. Sánchez, J. Ortega y F. Berbesi "Macroinvertebrados acuáticos como bioindicadores de la calidad del agua en zona media del Río Pamplonita,"
Cúcuta: UFPS. Colombia, pp. 35-40, 2001.

[5] M.Sánchez, "Elíndicebiológico BMWP (Biological Monitoring Working Party Score), modificado y adaptado al cauce principal del Río Pamplonita Norte de Santander", Bistua, vol. 3, no. 2, pp. 55, 2005 .

[6] M.J. Sánchez-Herrera y M.J. AvendañoSánchez, "Macroinvertebrados del Norte de Santander", Respuestas, vol. 10, no. 1, pp. 3-20, 2005.

[7] J.Y. Ortega, "Modelación y Simulación dinámica de Macroinvertebrados acuáticos como bioindicadores de la calidad del agua". Tesis Magister, Facultad de Ingeniería, Universidad de Pamplona, Colombia, 2012.

[8] Instituto de Hidrología, Meteorología y Estudios Ambientales. Guía para el monitoreo de vertimientos, aguas superficiales y subterráneas, 2002.

[9] J.H. Suarez y Y.E. Rodríguez. Laboratorio de Biotecnología Ambiental, Universidad Francisco de Paula Santander, 2008, última actualización 2015.

[10] Friends of Five Creeks. Water Quality Monitoring: Dissolved Oxygen. [En línea]. Disponible: http://www. fivecreeks.org/monitor/do.shtml.

$-820 \mathrm{X}$

ISSN 2422-5053

PP: $112-128$

[11] J.N Fernández, Índices de calidad y de contaminación del Agua, Pamplona: Centro de Publicaciones Universidad de Pamplona, 2005.

[12] A. Ramírez y G. Viña, "Limnología Colombiana" Bogotá D.C, pp. 108-126. 1999. 
No. 1

Enero - Junio 2017

ISSN 0122-820X

E-ISSN 2422-5053

PP: $112-128$

[14] N. Fernández, A. Ramírez y J.Y. Ortega, "Modelación dinámica de una comunidad de macroinverteberados bentónicos frente a un gradiente temporal de contaminación orgánica, propuesta metodológica", en VII semana de Ciencia, tecnología e innovación, UFPS, pp. 56-61, 2010.

[15] N. Fernández y F. Solano, "Índices de Calidad y de Contaminación del agua," Colombia, Pamplona, 2008.

[16] Normas técnicas de calidad del agua potable. Bogotá, Colombia, Decreto 475 art. 39, mar. 1998.

[17] A. Ramírez. "Ecología, métodos de muestreo y análisis de poblaciones y comunidades", Bogotá. D.C. p. 61, 2006. 